\title{
Relationship between Critical Shoulder Angle and Rotator Cuff Tears in Patient with Non-Traumatic Shoulder Pain
}

Dr. Deepak KS ${ }^{1}$, Dr. Bharat $\mathrm{MP}^{2 *}$

\section{${ }^{1,2}$ Associate Professor, Department of Rad \\ Article History \\ Received: 01.09.2020 \\ Accepted: 19.09 .2020 \\ Published: 19.12.2020 \\ Journal homepage: \\ https://www.easpublisher.com}

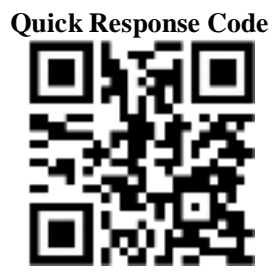

diodiagnosis, Subbaiah Institute of Medical Sciences, Shimoga, Karnataka, India

Abstract: Background: Aim of this study was to investigate the potential influence of Critical Shoulder Angle (CSA) as a predisposing factor for the development of degenerative full-thickness rotator cuff tears (DRCT) or primary glenohumeral osteoarthritis (PGOA). Materials and Methods: This was an observational and prospective study, with subjects presenting with pain and/or restricted motion of their shoulder/s (symptomatic shoulders) presenting to the orthopedic Department and Radiology, Subbaiah Institute of Medical Sciences conducted between December 2019 to August 2020. We divided the subjects into case and control groups on the basis of presence and absence of "non-traumatic RCTs," respectively, as per their USG (in three patients) and/or MRI shoulder scans. There were 60 study subjects and 60 control subjects. Results: The mean age of the control group was 56.37 years, while that of the study group was 58.42 years. The study group had a predominance of males $(58 \%)$. About $62 \%$ of subjects in the study group had right-sided symptomatic shoulders. The mean CSA of control subjects was $32.63^{\circ}\left( \pm 1.87^{\circ}\right)$, while that of study subjects were $36.72^{\circ}\left( \pm 2.24^{\circ}\right)$. The minimum and maximum CSA values in the study and control groups as well as their mean, median and mode, with standard deviation. Mean CSA values of study and control groups are represented. Conclusion: The CSA was an objective assessment tool to identify patients with shoulder pain who may have RCTs. Our study indicated that the CSA predicted RCTs more accurately than did the AI for patients with shoulder pain.

Keywords: Shoulder; rotator cuff tear; critical shoulder angle; acromial index.

Copyright (C) 2020 The Author(s): This is an open-access article distributed under the terms of the Creative Commons Attribution 4.0 International License (CC BY-NC 4.0) which permits unrestricted use, distribution, and reproduction in any medium for non-commercial use provided the original author and source are credited.

\section{INTRODUCTION}

A rotator cuff tear $(\mathrm{RCT})$ is a disorder characterized by shoulder pain and dysfunction. RCTs are common, found in approximately $15 \%-20 \%, 26 \%$ $30 \%$, and $36 \%-50 \%$ of people aged 60,70 , and 80 years, respectively $[1,2]$. Disorders of the rotator cuff account for $30 \%$ to $70 \%$ of cases of painful shoulders, and the prevalence of RCTs has been reported to be $22.1 \%$ in the general population [3]. Patients with RCTs can be asymptomatic, and this may have led to the varying prevalence among different studies. RCTs may be more prevalent than reported because of asymptomatic cases. Surgery is required for severe RCTs to restore shoulder function.

RCTs have multiple causes, which can be classified as intrinsic (degeneration, microtrauma, and hypoperfusion) or extrinsic factors (chronic impingement syndrome and overuse) [4]. Recently, anatomic factors, such as the critical shoulder angle (CSA) and acromion index (AI), have become key associated aspects of RCTs and the prognosis of repair surgery [5]. The CSA is the angle formed from the line of the superior to inferior bony margin of the glenoid and inferior bony margin of the glenoid to the lateral margin of the acromion. The AI is defined as the distance from the glenoid plane to the acromial lateral border, divided by the distance from the glenoid plane to the lateral aspect of the humeral head. The CSA can indicate glenoid inclination and lateral extension of the acromion. The AI reveals lateral extension of the acromion [6]. Previous studies have reported that a higher CSA and AI are associated with fullthickness RCTs [7, 8]. Another study indicated that patients with degenerative RCTs had a higher CSA than did those without RCTs [9].

Although the CSA and AI have been mentioned as radiographic assessment measures and are associated with RCTs, there are no relevant studies that have investigated their accuracy for predicting RCTs [10]. In addition, for clinical practice, early detection of RCTs is important for effective treatment. Therefore, we investigated the sensitivity and specificity of CSA and AI measurements for detecting patients with RCTs among a population with nontraumatic shoulder pain.

\section{RATiONALE OF THE STUDY}

A radiograph of the shoulder is the most common radiological investigation conducted in 
orthopedic outpatient departments. Despite giving the aforementioned indirect signs, a radiograph cannot characterize a RCT, which requires ultrasonography (USG) and/or magnetic resonance imaging (MRI). We aim to provide CSA determined on an anteroposterior (AP) shoulder radiograph, as an additional predictor, in the supplement to the clinical examination, for diagnosing RCTs, which can be further characterized in detail by MRI and USG.

\section{Materials ANd Methods}

This was an observational and prospective study, with subjects presenting with pain and/or restricted motion of their shoulder/s (symptomatic shoulders) presenting to the orthopedic Department and Radiology, Subbaiah Institute of Medical Sciences conducted between December 2019 to August 2020. We divided the subjects into case and control groups on the basis of presence and absence of "non-traumatic RCTs," respectively, as per their USG (in three patients) and/or MRI shoulder scans. There were 60 study subjects and 60 control subjects.

Inclusion criteria: For the case and control groups were symptomatic shoulders with no history of trauma and radiologically confirmed RCTs.

Exclusion criteria Patients with asymptomatic shoulder, history of trauma, glenohumeral osteoarthritis, and infections were excluded from the study.

After the informed consent was obtained, the epidemiological analysis sheet was completed for each subject. Following this, true AP shoulder radiographs of the subjects were evaluated. Accepted radiographs were those with $<20^{\circ}$ of internal or external rotation (those radiographs where upper and lower glenoid margins were clearly discernible), in accordance with the study of Moor et al., [11] The radiographs were evaluated by drawing a line connecting the upper (point X) and lower (point Y) glenoid margins. Another line was drawn from point $\mathrm{Y}$ to the lateral-most acromial projection (point Z). Angle XYZ was the CSA (Figure-1).

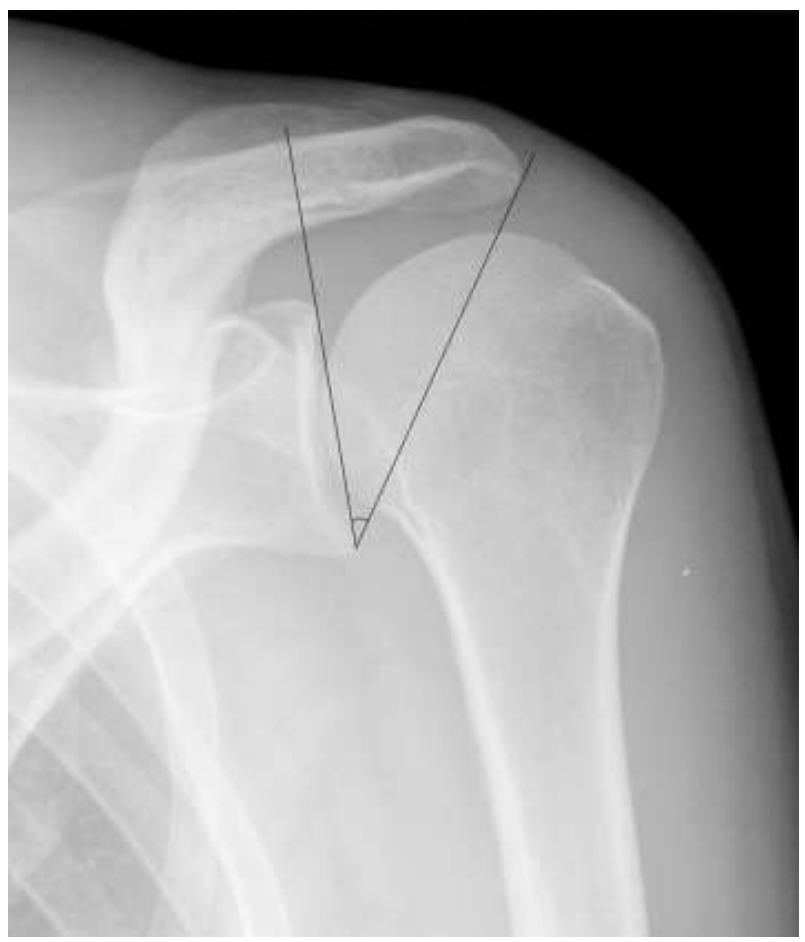

Fig-1: Critical shoulder angle

\section{Statistical Analysis}

Chi-square test applied to the distribution in Table-2 gave $\mathrm{P}=0.00001$, representing an association between the study groups and the CSA.

\section{RESULT}

The mean age of the control group was 56.37 years, while that of the study group was 58.42 years. The study group had a predominance of males (58\%). About $62 \%$ of subjects in the study group had right-sided symptomatic shoulders. Table-1 depicts the age, sex, and side distribution of the study and control groups.

Table-1: Age, sex, and side distribution of the study and control groups

\begin{tabular}{|l|l|l|}
\hline Parameter & Control Group $(\mathrm{n}=60)$ & Study Group $(\mathrm{n}=60)$ \\
\hline Mean Age & 56.53 years $(44-78)$ & 58.42 years $(44-78)$ \\
\hline Sex & \multicolumn{3}{|l|}{} \\
\hline Females & $30(50)$ & $25(42)$ \\
\hline Males & $30(50)$ & $35(58)$ \\
\hline Side & & $37(62)$ \\
\hline Right & $31(51.7)$ & $23(38)$ \\
\hline Left & $29(48.3)$ &
\end{tabular}

Table-2: Minimum and maximum CSA values in the study and control groups as well as their mean, median, and mode, with SD

\begin{tabular}{|l|l|l|l|l|l|l|}
\hline Subject & Min. CSA & Mean & Median & Mode & Max. CSA & SD \\
\hline Control & 29.7 & 32.63 & 32.43 & 32.3 & 38.2 & 3.61 \\
\hline Study & 32.7 & 36.72 & 36.83 & 37.4 & 48.7 & 5.06 \\
\hline
\end{tabular}

SD: Standard deviation, CSA: Critical shoulder angle 
The mean CSA of control subjects was $32.63^{\circ}$ $\left( \pm 1.87^{\circ}\right)$, while that of study subjects were $36.72^{\circ}$ $\left( \pm 2.24^{\circ}\right)$. Table- 2 shows the minimum and maximum CSA values in the study and control groups as well as their mean, median and mode, with standard deviation. Mean CSA values of study and control groups are represented.

Table-3: Group-wise distribution of study and control subjects having CSA values on either side of $34^{\circ}$

\begin{tabular}{|l|l|l|l|}
\hline Crossed table & Group 1 (with CSA $\leq \mathbf{3 4}^{\circ}$ ) & Group 2 (with CSA $>34^{\circ}$ ) & Total \\
\hline Control Group & 54 & 6 & 60 \\
\hline Study Group & 3 & 57 & 60 \\
\hline Total & 57 & 63 & 120 \\
\hline
\end{tabular}

CSA: Critical shoulder angle

In Table-3 we divided the subjects into two groups, as we noted there was a predominance of CSA values more than $34^{\circ}$ in the study subjects, while CSA values of $\leq 34^{\circ}$ were predominant in the control subjects. Subjects with CSA $\leq 34^{\circ}$ were put into Group 1, while those with CSA values more than $34^{\circ}$ were put in Table 3 . Hence, the CSA chosen was $34^{\circ}$

\section{Table-4: Distribution of rotator cuff lesions}

\begin{tabular}{|l|l|}
\hline RCLs in larger CSAs & No. (\%) \\
\hline Supraspinatus & $36(60 \%)$ \\
\hline Supraspinatus + Subscapularis & $12(20 \%)$ \\
\hline Supraspinatus + Infraspinatus & $9(15 \%)$ \\
\hline Subscapularis & $2(3.3 \%)$ \\
\hline Infraspinatus & $1(1.66 \%)$ \\
\hline
\end{tabular}

We also observed that most of the RCTs were those involving the supraspinatus tendon alone $(60 \%)$, followed by the combined affliction of supraspinatus and subscapularis tendons (20\%). Infraspinatus was more commonly affected along with the supraspinatus tendon $(15 \%)$, rather than being involved alone $(1.66 \%$ - in just one study subject). The distribution of different RCTs is depicted in Table-4.

The data tended to symmetry as the median and mean values for both study and control groups were relatively close. The relative variability in the control group was about $6 \%$ and that in the study group was also $6 \%$, indicating internal homogeneity of the study groups.

\section{DisCUSSION}

When patients visit hospital for shoulder pain, a radiographic evaluation is usually the initial assessment tool; some hospital are not equipped with ultrasound capabilities. Radiographic measurement parameters could help clinicians determine the requirement of a further ultrasound evaluation for patients with shoulder pain and RCTs. Our study revealed that compared with the AI, the CSA could predict RCTs for patients with shoulder pain with better accuracy. A high CSA indicated high acromial coverage and glenoid inclination and contributed to the vulnerability of RCTs from impingement. In a biomechanical study, Gerber et al evaluated and compared the joint reaction force of a CSA of more than $38^{\circ}$ with that of more than $33^{\circ}$ in patients with RCTs [12]. They reported that an increased CSA could lead to decreased superoinferior joint stability and that shoulder instability led to compensatory increasing loads on the supraspinatus tendon [13]. High-loaded supraspinatus tendons could incur repetitive shoulder active adduction, leading to overloading of rotator cuffs and causing tears [14].

The pathogenesis of RCTs is typically classified as having extrinsic (overuse, chronic impingement syndrome, and multifactorial causes) or intrinsic factors (degeneration, hypoperfusion, microtrauma, and apoptosis) [15]. The higher risk of RCTs among patients with a high CSA may be explained by extrinsic impingement and high tensile stress overloading. Previous studies have noted that tensile stress overloading on rotator cuff tendons is a pathogenesis of RCTs [16]. Gartsman GM et $a l$., reported that the CSA could contribute to the risk of degenerative RCTs by affecting glenoid inclination and lateral extension of the acromion [17]. Previous biomechanical studies have confirmed that a high CSA was associated with overloaded tensile stress on rotator cuff tendons, which then led to RCTs [18].

CSA as a parameter was first introduced by Moor et al., [11] Nyffeler et al., [15] introduced the acromion index (AI), suggesting an association between a greater lateral projection of the acromion and a possible rotator cuff injury. According to Armstrong would be the first to indicate impingement on supraspinatus tendon due to acromial morphology. ${ }^{[19]}$ Bigliani et al., identified different acromial morphologies [20]. Banas et al., also reported an association between the increased lateral acromial inclination and subacromial pathology [21].

Gerber et al., in 2014, proposed that CSA quantifies the lateral acromial coverage independently of features seen in glenohumeral osteoarthritis, such as humeral head flattening or bony erosions of glenoid [12]. He also performed a biomechanical analysis constructing a simulator and hypothesized that increased CSAs lead to an increase in joint shear to joint compression forces (instability ratio), which requires supraspinatus overloading, especially during initial stages of abduction. Our study observations 
represent an association between increased CSAs and non-traumatic RCTs. These findings are in sync with Suter T et al., [22].

Normal healthy shoulder is defined by a balanced mechanical overload [23, 24]. CSA essentially defined the acromial anatomic configuration, in that apart from reflecting the acromial coverage, it also indicates the glenoid the drawback of our study included being limited by the accuracy of USG and/or MRI in diagnosing the RCTs, as all the study subjects did not undergo surgery and hence arthroscopic confirmation of the tears.

\section{Conclusion}

Patients with RCTs presented a higher CSA than did patients without RCTs, and the CSA could be an objective assessment tool to predict RCTs for patients with shoulder pain. Furthermore, our study revealed that the CSA had better accuracy for predicting RCTs than did the AI for patients. When the CSA surpasses the cutoff value on radiographic assessments, further ultrasonography is recommended to detect RCTs. There is a significant direct association between larger CSA and non-traumatic RCTs.

\section{REFERENCES}

1. Blonna, D., Giani, A., Bellato, E., Mattei, L., Caló, M., Rossi, R., \& Castoldi, F. (2016). Predominance of the critical shoulder angle in the pathogenesis of degenerative diseases of the shoulder. Journal of shoulder and elbow surgery, 25(8), 1328-1336.

2. Gerber, C., Snedeker, J. G., Baumgartner, D., \& Viehöfer, A. F. (2014). Supraspinatus tendon load during abduction is dependent on the size of the critical shoulder angle: a biomechanical analysis. Journal of Orthopaedic Research, 32(7), 952-957.

3. Huang, S. W., Wang, W. T., Chou, L. C., Liou, T. H., Chen, Y. W., \& Lin, H. W. (2016). Diabetes mellitus increases the risk of rotator cuff tear repair surgery: a population-based cohort study. Journal of Diabetes and its Complications, 30(8), 1473-1477.

4. Huang, S. W., Wu, C. W., Lin, L. F., Liou, T. H., \& Lin, H. W. (2017). Gout can increase the risk of receiving rotator cuff tear repair surgery. The American Journal of Sports Medicine,45(10), 2355-2363.

5. Nyffeler, R. W., Werner, C. M., Sukthankar, A., Schmid, M. R., \& Gerber, C. (2006). Association of a large lateral extension of the acromion with rotator cuff tears. JBJS, 88(4), 800-805.

6. Chillemi, C., \& Franceschini, V. (2013). Shoulder osteoarthritis. Arthritis, 2013.; 2013:1-7.

7. Sambandam, S. N., Khanna, V., Gul, A., \& Mounasamy, V. (2015). Rotator cuff tears: An evidence based approach. World journal of orthopedics, 6(11), 902.

8. Park, H. B., Gwark, J. Y., Im, J. H., Jung, J., Na, J. B., \& Yoon, C. H. (2018). Factors associated with atraumatic posterosuperior rotator cuff tears. The Journal of Bone and Joint Surgery. American Volume, 100(16), 1397-1405.

9. Via, A. G., De Cupis, M., Spoliti, M., \& Oliva, F. (2013). Clinical and biological aspects of rotator cuff tears. Muscles, ligaments and tendons journal, 3(2), 70-79.

10. Fukuda, H. (2000). Partial-thickness rotator cuff tears: a modern view on Codman's classic. Journal of Shoulder and Elbow Surgery, 9(2), 163-168.

11. Moor, B. K., Bouaicha, S., Rothenfluh, D. A., Sukthankar, A., \& Gerber, C. (2013). Is there an association between the individual anatomy of the scapula and the development of rotator cuff tears or osteoarthritis of the glenohumeral joint? A radiological study of the critical shoulder angle. The bone \& joint journal, 95(7), 935-941.

12. Gerber, C., Snedeker, J. G., Baumgartner, D., \& Viehöfer, A. F. (2014). Supraspinatus tendon load during abduction is dependent on the size of the critical shoulder angle: a biomechanical analysis. Journal of Orthopaedic Research, 32(7), 952-957.

13. Lashgari, C. J., \& Yamaguchi, K. (2002). Natural history and nonsurgical treatment of rotator cuff disorders. In: Norris, T. R., editor. Orthopaedic knowledge update. Rosemont, IL: AAOS Shoulder and Elbow; 155-62.

14. Mileski, R. A., \& Snyder, S. J. (1998). Superior labral lesions in the shoulder: pathoanatomy and surgical management. JAAOS-Journal of the American Academy of Orthopaedic Surgeons, 6(2), 121-131.

15. Nyffeler, R. W., Werner, C. M., Sukthankar, A., Schmid, M. R., \& Gerber, C. (2006). Association of a large lateral extension of the acromion with rotator cuff tears. JBJS, 88(4), 800-805.

16. Neer, C. S. (1990). Anatomy of shoulder reconstruction. In: Neer, C. S., editor. Shoulder reconstruction. Philadelphia: W. B. Saunders Company; 1-39.

17. Gartsman, G. M., \& Milne, J. C. (1995). Articular surface partial-thickness rotator cuff tears. Journal of Shoulder and Elbow Surgery, 4(6), 409-415.

18. Walch, G., \& Liotard, J. P. (1992). Les lesions tendineuses de l'épaule. In: Saillant, G., Catonne, Y., editors. Lesions traumatiques des tendons chez le sportif. Paris: Masson; 1-23.

19. Armstrong, J. R. (1949). Excision of the acromion in treatment of the supraspinatus syndrome. The Journal of Bone and Joint Surgery. British volume, 31(3), 436-442.

20. Bigliani, L. U. (1986). The morphology of the acromion and its relationship to rotator cuff tears. Orthop trans, 10, 216-228. 
21. Banas, M. P., Miller, R. J., \& Totterman, S. (1995). Relationship between the lateral acromion angle and rotator cuff disease. Journal of Shoulder and Elbow Surgery, 4(6), 454-461.

22. Suter, T., Popp, A. G., Zhang, Y., Zhang, C., Tashjian, R. Z., \& Henninger, H. B. (2015). The influence of radiographic viewing perspective and demographics on the critical shoulder angle. Journal of Shoulder and Elbow Surgery, 24(6), e149-e158.
23. Dunteman, R., Fukuda, H., \& Snyder, S. J. (2002). Surgical treatment of partial-thickness tears. Orthopaedic knowledge update: shoulder and elbow, 2, 163-170.

24. Spiegl, U. J., Horan, M. P., Smith, S. W., Ho, C. P., \& Millett, P. J. (2016). The critical shoulder angle is associated with rotator cuff tears and shoulder osteoarthritis and is better assessed with radiographs over MRI. Knee Surgery, Sports Traumatology, Arthroscopy, 24(7), 2244-2251. 